\title{
Real-time Measurement of Protein Crystal Growth Rates within the Microfluidic Device to Understand the Microspace Effect
}

Masatoshi Maeki, ${ }^{*}$ Shohei Yamazaki, ${ }^{b}$ Reo Takeda, ${ }^{b}$ Akihiko Ishida, ${ }^{a}$

Hirofumi Tani, ${ }^{a}$ and Manabu Tokeshi ${ }^{a^{*}}$

aivision of Applied Chemistry, Faculty of Engineering, Hokkaido University, Kita 13 Nishi 8, Kita-ku, Sapporo 060-8628, Japan

${ }^{b}$ Graduate School of Chemical Sciences and Engineering, Hokkaido University, Kita 13 Nishi 8, Kita-ku, Sapporo 060-8628, Japan 


\section{Raman spectrum of the microfluidic device substrate}

Figure S1 shows a comparison of the Raman spectra of a lysozyme crystal prepared at the $40 \mathrm{mg} / \mathrm{mL}$ lysozyme and $0.7 \mathrm{M} \mathrm{NaCl}$ condition, 70- $\mu \mathrm{m}$-thick polydimethylsiloxane (PDMS) film, and 50- $\mu \mathrm{m}$-thick cyclic olefin polymer (COP) film. We observed high Raman intensity from the lysozyme crystal at $2940 \mathrm{~cm}^{-1}$. However, PDMS and COP films also showed Raman shifts at $2940 \mathrm{~cm}^{-1}$. In contrast, glass substrate did not show any Raman shift at $2940 \mathrm{~cm}^{-1}$ (Figure S2 [air]). Thus, we prepared the microwell using a PDMS substrate sandwiched between glass substrates.

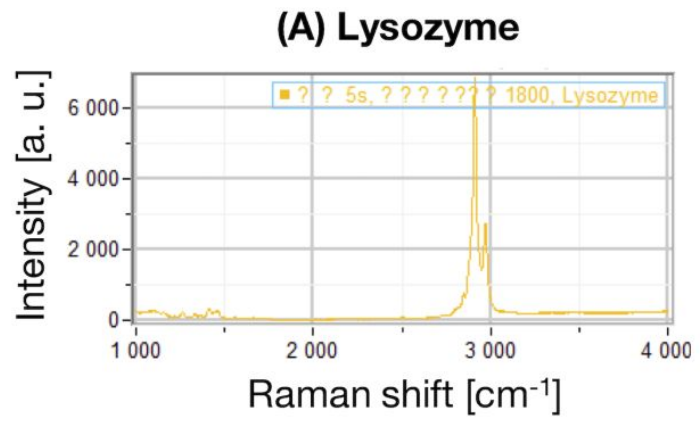

(B) $70 \mu \mathrm{m}$ PDMS film

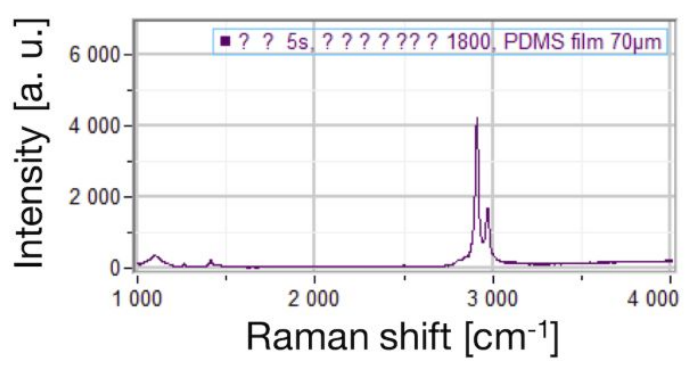

(C) $50 \mu \mathrm{m}$ COP film

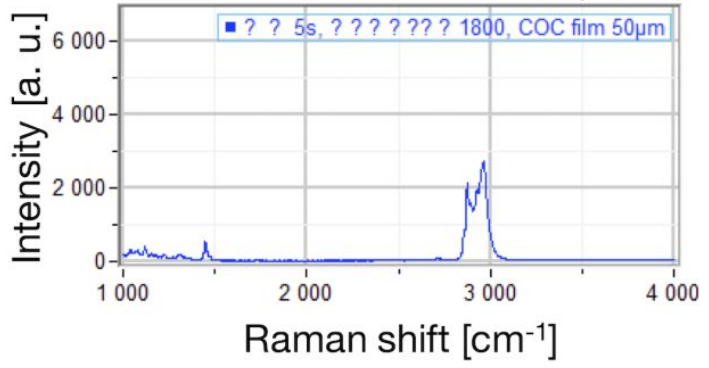

Figure S1. Raman spectra of the lysozyme crystal, polydimethylsiloxane (PDMS) film, and cyclic olefin polymer $(\mathrm{COP})$ film. 


\section{Comparison of the Raman intensities}

Figure S2 shows a comparison of the Raman intensities of a lysozyme crystal, lysozyme solution (40 mg/mL lysozyme and $0.7 \mathrm{M} \mathrm{NaCl})$, and air after incubation for $4 \mathrm{hrs}$. The lysozyme crystal exhibited the highest Raman intensity of the three, while air did not show any specific Raman shift. For these reasons, the Raman spectrum of air was used as a baseline spectrum to produce a protein concentration profile based on Raman mapping. Figure 3 represents the time sequence of the Raman mapping images from 4 hrs to $8 \mathrm{hrs}$.

(A)

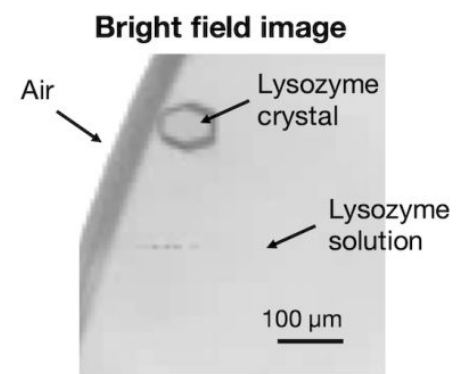

(B)
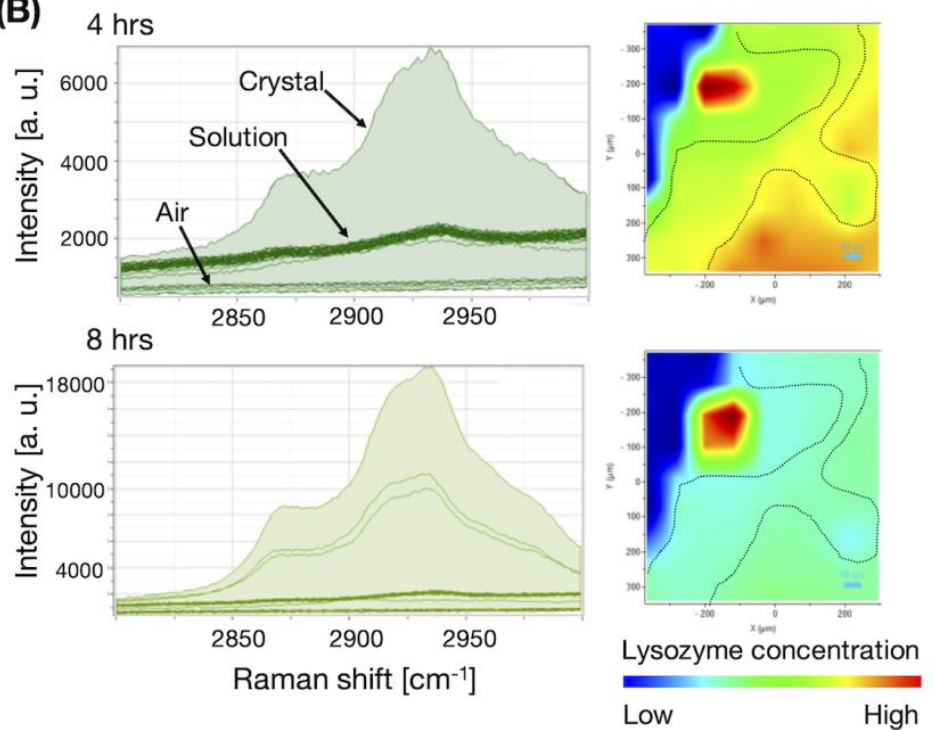

Figure S2. (A) Photograph of a lysozyme crystal. (B) (left) Raman spectra collected from the crystal, lysozyme solution, and air, and (right) Raman mapping images after incubation for 4 hrs and 8 hrs. 

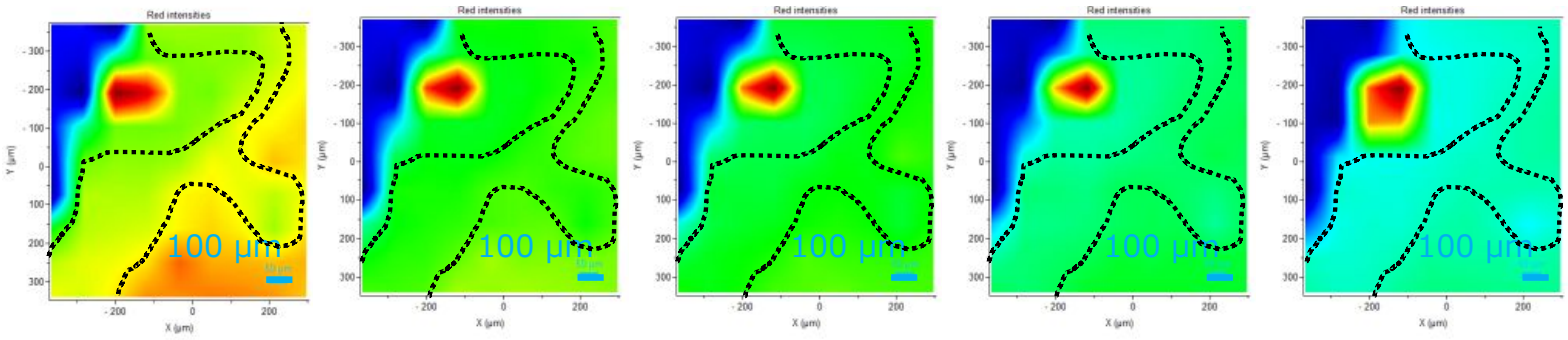

Figure S3. Time sequence of the Raman mapping images from 4 hrs to 8 hrs. 